\title{
Trauma der Nebenniere - bilaterale adrenale Hämorrhagie
}

Die bilaterale adrenale Hämorrhagie (BAH) nach stumpfem Bauchtrauma ist extrem selten. Eine retrospektive Analyse von Computertomografien (CT) bei stumpfen Bauchtraumen weist eine Inzidenz adrenaler Blutungen von 0,03\% aus (Burks et al., Am J Roentgenol 1992; 58: 503). Da die Nebennieren aufgrund ihrer tiefen anatomischen Lage und geringen Größe relativ gut geschützt sind, liegen bei ihrer Verletzung zumeist Läsionen anderer gleichseitiger Abdominalorgane vor. Die BAH ist häufiger Folge nichttraumatischer Ursachen (z.B. Koagulopathie).

\section{Fallbeschreibung}

Ein 41jähriger Mann ohne anamnestische Besonderheiten wurde beim Überqueren der Straße von einer Straßenbahn mit geringer Geschwindigkeit erfasst. Bei dem initial bewusstseinsklaren, hämodynamisch stabilen Patienten erfolgte wenig später aufgrund von Somnolenz und respiratorischer Insuffizienz die Intubation und Beatmung.

Klinisch bestanden Hinterkopf- und Ellenbogenlazerationen, klavikuläre Krepitationen sowie eine Flankenkontusion rechts. Die Bauchdecken waren weich.

Als initiale bildgebende Diagnostik wurde eine Sonografie (Siemens Sonoline, 3,5 MHz) im Schockraum durchgeführt (Abb.1). In der Loge der rechten Nebenniere kam eine umschriebene echoarme Raumforderung $(45.26 \mathrm{~mm})$ zur Darstellung. Links war die Umgebung des kranialen Nierenpoles bei eingeschränkter Beurteilbarkeit diffus echoarm. Die Befunde in den Nebennierenlogen konnten sonografisch nicht sicher interpretiert werden. Im Anschluss erfolgte eine Spiral-CT (Philips Tomoscan SR 7000) des Abdomens (Abb. 2). Hier fanden sich bilateral hyperdense Raumforderungen $(52-55 \mathrm{HE})$ in beiden Nebennierenlogen (50.34 mm rechts; $41.30 \mathrm{~mm}$ links). Regelrechte adrenale Strukturen waren nicht vorhanden. Das periadrenale und

Fortschr Röntgenstr 2000; 172: 946-947 (c) Georg Thieme Verlag Stuttgart · New York ISSN 1438-9029

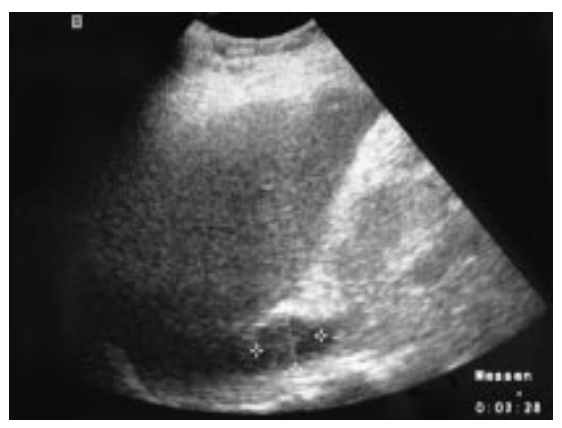

Abb. 1 Sonografie (3,5 MHz). Echoarme subhepatische Raumforderung in der rechten Nebennierenloge - posttraumatisches adrenales Hämatom.

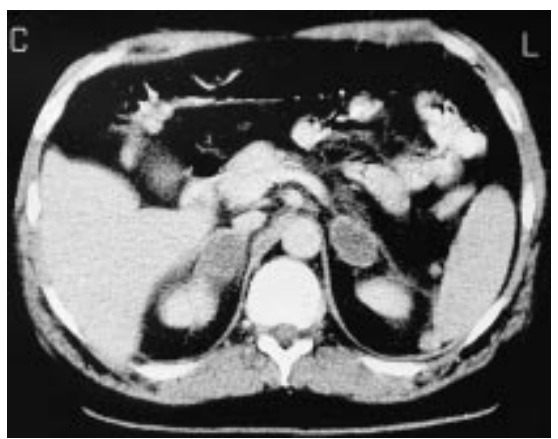

Abb. 2 Spiral-CT (KM i.v., Schichtkollimation/Tischvorschub/Inkrement - 10/10/8). Akutes hyperdenses Hämatom (52-55 HE) beider Nebennieren, saumartiges Randenhancement - komprimiertes, intaktes Nebennierengewebe. Feine periadrenale Hämorrhagien, keine Ruptur.

kraniale perirenale Fettgewebe war z.T. streifig densitätserhöht und maskiert. In beiden Raumforderungen zeigte sich randständig ein schmaler anreichernder Gewebssaum. Es handelte sich um bilaterale adrenale Hämatome mit Kompression und saumartiger Verlagerung intakten Nebennierengewebes an den Hämatomrand sowie begleitende feine Hämorrhagien im periadrenalen Fettgewebe. Der rechte Leberlappen wies zusätzlich einen kleinen Kontusionsbezirk auf.

Weitere Verletzungen waren eine Rippenserienfraktur rechts mit Hämatothorax und Lungenkontusion sowie Klavikulafrakturen beidseits.
Nach Stabilisierung, Anlage einer Thoraxsaugdrainage, Bronchiallavage und Wundversorgung erfolgte die Verlegung auf die chirurgische Intensivstation. Am zweiten Tag entwickelte der Patient eine Bronchopneumonie, die therapeutisch beherrscht werden konnte.

Klinisch und laborchemisch (Serumhormone, 24-Stunden-Urin) lagen zu keinem Zeitpunkt Zeichen der adrenalen Insuffizienz wie z. B. Elektrolytdysbalance oder Hypotonie vor. Nach drei Wochen erfolgte die Verlegung auf die Normalstation und nach einer weiteren Woche die Entlassung.

Drei Wochen nach dem Trauma wurde eine Magnetresonanztomografie der Nebennieren (MRT, Siemens Magnetom Vision 1,5 T) zur Verlaufskontrolle und zum Ausschluss einer blutungsbegünstigenden Pathologie (z.B. eines Nebennierentumors) durchgeführt (Abb.3). Die Signalcharakteristika - $\mathrm{T}_{1}$-gewichtet signalarm (Gradienten-Echo-Sequenz FLASH 2D), $\mathrm{T}_{2}$-gewichtet inhomogen signalreich/-arm (Turbo-Spin-Echo-Sequenz - TSE) - entsprachen beidseitigen adrenalen Hämatomen im späten subakuten Stadium mit verschiedenen Blutabbauprodukten (extrazelluläres Methämoglobin, ebenso Ferritin/Hämosiderin). Auf i.v. Kontrastmittel musste patientenbedingt verzichtet werden. Da ein adrenaler hämatomüberlagerter Tumor letztlich nicht auszuschließen war, wurde eine Follow Up-Untersuchung angeraten.

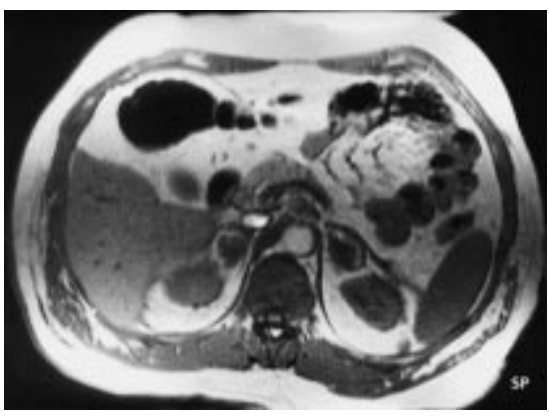

Abb. 3 MRT (nativ, $\mathrm{T}_{1} \mathrm{w}$ FLASH 2D). Hypointense Raumforderung, partiell hyperintenser Randsaum - bilaterales adrenales Hämatom im späten subakuten Stadium. Schlanker medialer Schenkel rechts.

15 Monate nach dem Unfall, in denen keine Symptome einer Nebennierenfunktionsstörung aufgetreten waren, erfolgte die abschließende Untersuchung. 
Die Spiral-CT zeigte jetzt deutlich kleinere, partiell noch deformierte Nebennieren mit vereinzelten Mikroverkalkungen. Magnetresonanztomografisch $(1,5 \mathrm{~T})$ (Abb. 4) fanden sich in $\mathrm{T}_{1}$ - und $\mathrm{T}_{2}$-Wichtung deutlich signalarme, z.T. plumpe Nebennieren mit Suszeptibilitätsartefakten. Dieses Signal war bedingt durch organisierte Hämatome mit Hämosiderin, Kalzifikationen und Fibrosierungen. Es ergab sich kein Anhalt für einen Nebennierentumor.

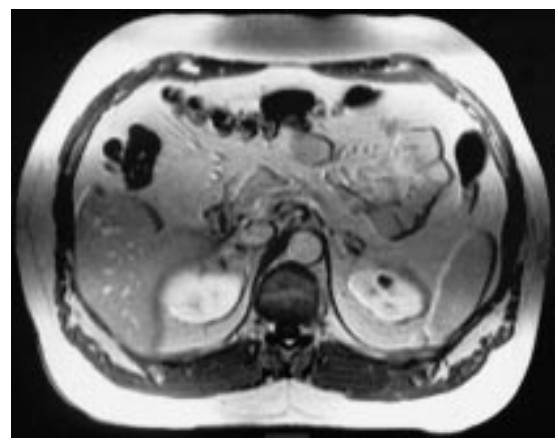

Abb. 4 MRT (Gad-DTPA i.v., T, w FLASH 2D). Noch partielle Verplumpung, parenchymales Enhancement. Suszeptibilitätseffekte als Ausdruck von Hämosiderin und Kalzifikationen.

\section{Diskussion}

Die Nebennieren scheinen besonders vulnerabel gegenüber Scherkräften zu sein, da Blutungen gelegentlich im Rahmen eines Geburtstraumas berichtet werden. Die Ursachen der Nebennierenblutung sind nicht exakt geklärt.

Folgende Verletzungsmechanismen sind anzunehmen (Gomez RG et al., J Trauma 1993; 35: 870; Ikekpeazzu N et al., J Emerg Med.1996; 14: 15; Murphy B et al., Radiology 1988; 169: 701):

1. Kompression der Drüse gegen die Wirbelsäule mit konsekutiver Organruptur

2. Intraadrenale Druckerhöhung infolge Kompression des venösen Abflusses.

3. Ruptur kleiner intraadrenaler Gefäße bedingt durch direkte Druck- und Scherwirkung.

Bei Entwicklung eines Hämatoms mit intraadrenaler Druckerhöhung thrombosieren kleine Gefäße mit dem Resultat der Gewebsischämie, -infarzierung und letztlich -nekrose (Feuerstein BP et al., Ann Intern Medic 1991; 115: 785). Im Verlauf kann eine adrenale Dysfunktion bzw. Insuffizienz auftreten. Nach schwerem Trauma mit Schock kann die Insuffi- zienz als Komplikation der Blutung, jedoch auch als Folge des kritischen Allgemeinzustandes des Patienten manifest werden. Der MCTH-Test, der die Insuffizienz und deren Grad nachweisen kann, ist jedoch bei Patienten mit beginnendem oder manifestem Schock praktisch nicht anwendbar.

Die klinischen Zeichen der BAH sind unspezifisch. Häufig sind abdominelle Schmerzen, Fieber und Hypotonie. Bei mehrfach verletzten Patienten ist keines dieser Zeichen spezifisch für die Diagnose. Die Sonografie im Schockraum ist nutzbringend, um bei Nachweis einer echoarmen Raumforderung am kranialen Nierenpol zumindest den Verdacht auf ein Nebennierenhämatom frühzeitig $\mathrm{zu}$ erheben (Abb.1). Die sichere radiologische Diagnose wird, wie in nahezu allen publizierten Fällen, letztlich mit der CT gestellt. Sie ermöglicht die Differenzierung zwischen dem organbeschränkten Hämatom und der klinisch noch nicht manifesten Organruptur. Zur Detektion einer Blutungsquelle und sicheren Evaluierung der übrigen Abdominalorgane ist die i.v. Kontrastmittelapplikation obligat.

Da die Gesamtprognose nach Nebennierenblutung stark von dem Zeitpunkt des Therapiebeginnes abhängt, kommt einer suffizienten Diagnostik in der Frühphase besonders bei polytraumatisierten Patienten, eine große Bedeutung zu. Hierin ist die Herausforderung für den Diagnostiker zu sehen. Während bei dem isolierten adrenalen Hämatom ein konservatives Vorgehen unter intensivmedizinischem Monitoring und eventueller Hormonsubstitution möglich ist, stellt die Organruptur eine absolute, dringliche Operationsindikation dar. Mehrzeitige letale Nebennierenrupturen können noch Tage nach Ausbildung des Hämatomes auftreten.

Im Rahmen von Multiorganverletzungen können der Evaluierung mit bildgebenden Verfahren Grenzen gesetzt sein. Bei der Nebennierenruptur kann ein ausgeprägtes retroperitoneales Hämatom, vor allem wenn es zur Verlagerung oder Kompression der Niere führt, möglicherweise nicht mehr sicher von einer Nierenverletzung unterschieden werden. Ein renal bedingtes Hämatom kann vice versa eine Nebennierenverletzung maskieren. $\mathrm{Zu}$ berücksichtigen ist, dass adrenale häufig mit renalen, aber auch hepatischen Verletzungen assoziiert sind.
Die MRT erbringt initial keine zusätzlichen Informationen, welche Änderungen des therapeutischen Regimes zur Folge hätten, und spielt somit in der Akutdiagnostik keine Rolle. Residuen eines klinisch okkulten Hämatomes können mit hoher Sensitivität unter Verwendung von Gradientenechosequenzen anhand von Suszeptibilitätseffekten nachgewiesen werden.

Da insbesondere kleine, im Nebennierenhämatom verborgene adrenale Tumoren der initialen Diagnostik entgehen können, sind nach adrenalen Hämatomen Verlaufsuntersuchungen zum Ausschluss derartiger prädisponierender Pathologien angezeigt.

\section{"Springende Punkte“ - Nebennierenverletzung}

- klinische Symptomatik häufig unspezifisch

- sichere Akutdiagnostik mittels CT

- intravenöse Kontrastmittelapplikation unabdinglich

- Differenzierung Hämatom/Ruptur wichtig für Therapie und Outcome

D. Mucha, S. Rammelt, Dresden

\section{Corrigendum}

In der Arbeit W. Kratzer et al.: Kontrastverstärkte Power-Doppler-Sonographie... in RöFo Mai 2000 (Fortschr Röntgenstr 2000; 172: $443-448$ ) wurden aufgrund eines Berechnungsfehlers falsche Werte gedruckt. Es handelt sich um die Mittelwerte und Mediane für die intra- und extratumorale Verstärkungsdauer mit der Bolusinjektion über den Hauptkanal in Tabelle 2, Seite 445. Betroffen sind auch die Werte auf Seite 445, rechte Spalte, 1. Absatz und in der Zusammenfassung auf Seite 443.

Die richtigen Werte lauten wie folgt:

Bolus extratumoral:

Mittelwert 5:55 min (statt 6:01)

Median 5:16 min (statt 5:35)

Standardabweichung 1:24 min (statt 1:30)

Bolus intratumoral:

Mittelwert 4:43 min (statt 4:54)

Median 4:37 min (statt 5:01)

Standardabweichung 1:49 min (statt 1:55) 